\title{
A new light-responsive resistive random-access memory device containing hydrogen-bonded complexes.
}

Thamil Selvi Velayutham ${ }^{\mathrm{a}, \mathrm{b}, \dagger}$, Nur M.S. Azmina ${ }^{\mathrm{a}}$ Vijayan Manickam-Achari ${ }^{\mathrm{b}, \mathrm{c}}$, Alejandro Roche $^{d}$, Rinaa Ramesh ${ }^{c}$, Alfonso Martinez-Felipe ${ }^{\mathrm{e}, \dagger}$

a Low Dimensional Materials Research Center, Department of Physics, Faculty of Science, University of Malaya, 50603 Kuala Lumpur, Malaysia.

${ }^{b}$ Centre for Fundamental and Frontier Sciences in Nanostructure Self-Assembly, Department of Chemistry, Faculty of Science, University of Malaya, 50603 Kuala Lumpur, Malaysia.

${ }^{c}$ Bioinformatics Program, Institute of Biological Sciences, Faculty of Science, University of Malaya, 50603 Kuala Lumpur, Malaysia.

` Departamento de Química Orgánica, Instituto de Ciencia de Materiales de Aragón, Universidad de Zaragoza-CSIC, Pedro Cerbuna 12, 50009 Zaragoza, Spain.

${ }^{e}$ Chemical and Materials Engineering Research Group, School of Engineering, University of Aberdeen, King's College, Old Aberdeen AB24 3UE, UK.

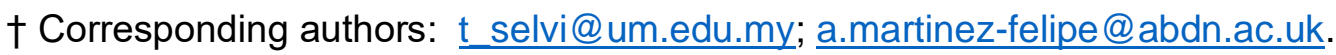




\section{Abstract}

In the search to obtain new and more efficient components of memory devices, we report the photochromic, dielectric and electrochemical response of a light-responsive organic compound, and its memory performance under electrical fields. The so-called N(1)-[12-(4(4'-isobutyloxyphenyldiazo)phenoxy)dodecyloxy)]thymine, tAZOi, molecule contains one azobenzene group, which provides with photochromic character, and one terminal thymine group, capable to form hydrogen bonds and assemble supramolecular dimers, (tAZOi) 2 . We have calculated the optical absorption coefficient, extinction coefficient and refractive index of tAZOi, which obeys the single oscillator Wemple-DiDomenico model. An ITO/tAZOi/AI device has been prepared and presents two switchable conductance states with preservation of memory performance. The mechanism linked to the resistive randomaccess memory (RRAM) has been evaluated by molecular modelling and is controlled by $p$ type conduction, possibly involving hydrogen-bonding. Upon UV irradiation at $\sim 365 \mathrm{~nm}$, tAZOi displays an increase in the complex permittivity driven by trans-to-cis ( $E$-to- $Z$ ) isomerisation of the azobenzene groups. Molecular simulations suggest that conductivity and device performance can be enhanced (and controlled) by light exposure through the formation of activated $Z$ isomers that could transfer charge to other neighbouring molecules, resulting in photo-electric responsive devices. 


\section{Introduction}

Photochromic molecules experience reversible photo-triggered isomerisation between two metastable states and are used as light-activated switches in different applications. A wide variety of photochromic materials exists undergoing valence isomerisations, $E / Z$ isomerisations, cycloadditions or tautomerisations, among others. The photochromic processes can involve pericyclic reactions (spiropyrans/-oxazine, diarylethenes), $E / Z$ isomerisations (azobenzenes, dithienylethene, stilbenes), photo-induced bond cleavages (triarylmethanes, perchlorotoulenes), intramolecular hydrogen-bond transfer (anils and polycyclic quinones) and electron transfer (viologens) [1-5].

Among these, azobenzene has been widely investigated as a photochromic group in lightresponsive materials due to its rapid, reversible and high quantum yield photo-isomerisation between $E$ and $Z$ conformations [6-9]. The azobenzene $E$ conformer (trans, ground state) is highly linear, whilst UV-vis illumination around $365 \mathrm{~nm}$ promotes isomerisation to a more curved Z conformer (cis, excited state), see Fig. 1. The resulting change in molecular geometry is very useful to prepare electrooptical devices based on the formation and disruption of anisotropic liquid crystalline phases [10-17]. This potential to exert temporal and spatial control over molecular order by light, also makes azobenzene an attractive component in controlled transport applications, including formulations for controlled release [13] or electrolytes for ionic conductivity [7,19-21].

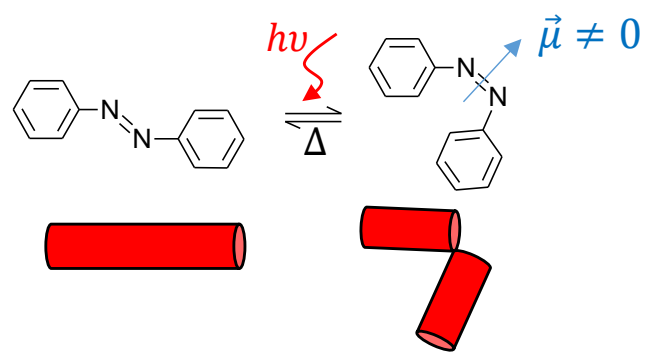

Fig. 1. Azobenzene $E$ (trans, left) and $Z$ (cis, right) isomers, showing the $E$-to- $Z$ isomerisation by UV-vis light irradiation, the thermally activated $Z$-to- $E$ relaxation, and the dipole moment in the $Z$ isomer, $\vec{\mu}$.

Azobenzenes have great potential as components of organic memory devices, due to their good charge transport capability via the $p-\pi$ backbone [22,23], and have been extensively used for data storage [24-28]. In order to avoid costly synthetic steps associated with the presence of the bulky azobenzene groups, hydrogen-bonding stands as an efficient strategy to yield new functional azo-materials [29-31]. Recently, we reported the synthesis and properties of a series of supramolecular polymers containing diaminopyridine units, DAP, to which azobenzene-containing molecules were attached via hydrogen-bonding [18]. We then 
assessed, by Fourier-transform infrared spectroscopy, FT-IR, that these azo-derivatives form supramolecular dimers [32], whose linearity can be useful to design novel anisotropic memory devices.

In this work, we explore the photochromic, dielectric and electrochemical response of one of the azo-derivatives developed in [18], the N(1)-[12-(4-(4'isobutyloxyphenyldiazo)phenoxy)dodecyloxy)]thymine, tAZOi, $\mathbf{1}$,<smiles>CCCCCCCCCCCCCCCn1cc(C)c(=O)[nH]c1=O</smiles>

\section{1, tAZOi}

Based on the application and availability of a great variety of transparent metal oxide-based switching media [33,34], resistive switching memories have distinguished as leading candidates for transparent and flexible information storage devices, using two-terminal metal-insulator-metal configurations $[35,36]$. The fabrication of resistive random-access memory devices, RRAM, with both high mechanical flexibility and good optical transparency, however, remains a challenge. In this work, we have evaluated the potential of tAZOi as a component in RRAM, and we believe that its performance will inspire the application of new and more efficient organic memory devices.

\section{Materials and experimental methods}

\subsection{Preparation and structural characterisation of tAZOi}

The synthesis of tAZOi has been reported previously, and details of its preparation and chemical characterisation can be found in [18]. tAZOi is a crystalline solid at room temperature, and melts into an isotropic phase at $166^{\circ} \mathrm{C}$. We did not find evidence of the formation of liquid crystalline phases [32], which must be hindered, at least in part, by the presence of the bulky isobutyloxy- terminations that disrupt the anisotropic interactions between azobenzenes [29].

The formation of hydrogen bonds was assessed by temperature-variable Fourier-transform infrared spectroscopy, FT-IR [19], using a Thermo Nicolet 470 FT-IR spectrometer over a frequency range of $4000-400 \mathrm{~cm}^{-1}$, with an accuracy of $4 \mathrm{~cm}^{-1}$, as the average of 64 scans. The measurement was carried out by measuring the IR transmittance through a disc containing a solid dispersion of tAZOi in potassium bromide, $\mathrm{KBr}(1 \%$ in weight \%), using one pristine $\mathrm{KBr}$ disc as the background. Temperature was controlled by placing the disc into a Linkam TMS93 heating stage, with a temperature accuracy of $\pm 0.1^{\circ} \mathrm{C}$. The sample 
was heated above its melting point and then cooled down to room temperature. The FT-IR spectra were then measured on isothermal steps every $10^{\circ} \mathrm{C}$, allowing the sample to equilibrate at each temperature during two minutes before measuring. X-ray diffractograms, XRD, of tAZOi were obtained using an evacuated Pinhole camera (Anton-Paar) operating with a point-focused Ni-filtered $\mathrm{Cu}-\mathrm{K}$ beam. The patterns were collected on flat photographic films perpendicular to the X-ray beam. A powdered tAZOi sample was placed into a quartz Lindemann capillary ( $0.7 \mathrm{~mm}$ diameter), and measured at room temperature.

In order to study the photochromic character of tAZOi, a certain amount of this compound was dissolved in tetrahydrofuran (THF) to obtain a $5 \mathrm{wt} \%$ solution, which was spin coated on a quartz substrate. The thickness of the resulting tAZOi film was $\sim 400 \mathrm{~nm}$. The ultraviolet-visible (UV-vis) absorbance $(A)$ and reflectance $(R)$ spectra were then measured directly from a Perkin Elmer Lambda 750UV-VIS-NIR spectrometer, in the 300 to $800 \mathrm{~nm}$ wavelength range. The film was measured before (ground state) and immediately after (excited state) being exposed for 10 minutes with UV light ( $\lambda=365 \mathrm{~nm}$, intensity $=50$ $\left.\mu \mathrm{m} \cdot \mathrm{cm}^{-2}\right)$. The sample was then kept in the dark, and was measured at various intervals until the spectra recovered its original shape (in order to monitor the thermal relaxation process).

The electrochemical properties of tAZOi were investigated by cyclic voltammetry (CV), using a Versa STAT 3 potentiostat in $0.1 \mathrm{M}$ of potassium chloride $(\mathrm{KCl})$ solution at room temperature. $1 \mathrm{mM}$ potassium ferricyanide was used as a supporting electrolyte in $\mathrm{KCl}$ solution, with ITO as the working electrode, a platinum gauze electrode as a counter electrode, and $\mathrm{Ag} / \mathrm{AgCl}$ as a reference electrode. The $\mathrm{CV}$ measurement was carried out at a sweep rate of 10 to $100 \mathrm{mV} \cdot \mathrm{s}^{-1}$ scan rate.

\subsection{Fabrication of memory device: dielectric and current-voltage (I-V) measurement}

A pre-patterned indium tin oxide (ITO) coated glass substrate $\left(\sim 10 \mathrm{ohm} \cdot \mathrm{sq}^{-1}\right.$ sheet resistance) was cleaned with soap water, acetone, isopropanol and distilled water, under ultra-sonication for 10 min each, and finally dried with nitrogen gas. A thin film of tAZOi was spin coated onto the ITO pre-cleaned substrate, and subsequently, a layer of aluminium (AI, thickness $\sim 60 \mathrm{~nm}$ ) was thermally deposited on top of the film under vacuum. The resulting memory device has 6 cells, and each cell contains $0.045 \mathrm{~cm}^{2}$ of photo-active area of a single photodetector, with a tAZOi film of $400 \pm 1 \mathrm{~nm}$ thickness measured with a KLA Tencor Surface Profilometer.

The performance of the memory device was tested under ambient conditions. The dielectric response of the device was studied using an impedance analyser (Agilent 4294A) in the frequency range from $40 \mathrm{~Hz}$ to $1.5 \mathrm{MHz}$, in the absence and presence of UV light $(\lambda=365$ 
$\mathrm{nm}$, intensity $\left.=50 \mu \mathrm{W} \cdot \mathrm{cm}^{-2}\right)$, respectively. The memory effect was then evaluated by obtaining current-voltage curves $(I-V)$, using a dual-channel system source meter (Keithley 2612B), and bias voltage was applied to the top metal electrode with respect to the ITO substrate.

\subsection{Density functional theory calculations (DFT)}

One single molecule of tAZOi was modelled using ChemBio2D, and loaded into ChemBio3D to have three dimensional Cartesian coordinates for the atomic positions. The energy of the structure was minimised by applying MMFF94 force field with the steepest descent method using Avogadro 31. The resulting minimised structure was loaded into GaussView 5.0 to generate an input file in gjf file format that was further optimised in Gaussian09 [37].

The tAZOi structure was geometrically optimised by applying analytic gradient techniques, using the density functional theory with Becke's three parameter exchange functional along with the Lee-Yang-Parr non-local correlation functional. The preliminary optimisation uses Hatree Fock level of theory with $3-21+G$ basis set and increases gradually following $6-31+G$, $6-31+G(d, p), 6-311+G$ and $6-311+G(d, p)$ basis set with B3LYP level of theory. The structure was further optimised with higher level CAM theory, which is a long-rangecorrected version of B3LYP using the coulomb-attenuating method by Handy and coworkers [38]. All the calculations were carried out in gas phase to mimic the system under dry conditions. Through these calculations, an electrostatic potential map was generated and visualised using the Avogadro software. Electrostatics potential maps were used to envisage the electron distribution on the molecular surfaces.

\section{Results and discussion}

\subsection{Hydrogen-bonding assessment, FT-IR}

The monomeric units of tAZOi can form multiple hydrogen bonds via the terminal thymine head, and their presence and strength can be assessed by Fourier-transform infrared spectroscopy, FT-IR. Fig. 2 shows the FT-IR spectra of tAZOi obtained on cooling from the isotropic phase $\left(T=170^{\circ} \mathrm{C}\right)$ to the crystal phase $\left(T=50^{\circ} \mathrm{C}\right)$, corresponding to the carbonyl stretching region, $\mathrm{C}=\mathrm{O}$ st. The appearance of several bands in the frequency range 1740 to $1650 \mathrm{~cm}^{-1}$ indicates that the carbonyl groups are present in a variety of intermolecular environments, via the formation of hydrogen bonds that yield different supramolecular assemblies [32]. 
(a)

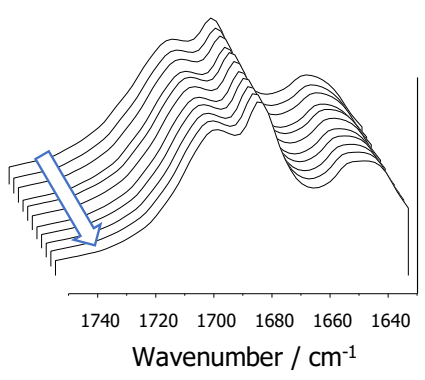

(b)

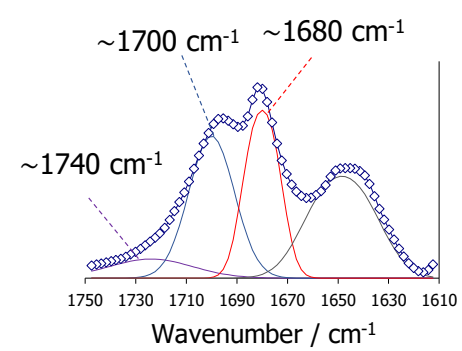

(c)

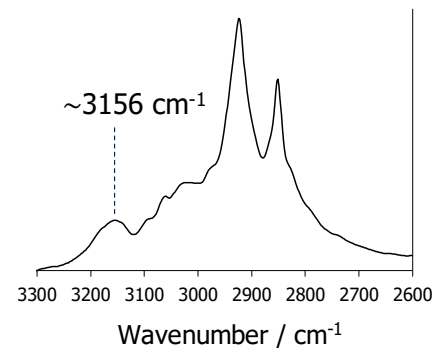

Fig. 2. (a) Variable-temperature FT-IR spectra for $t A Z O i$ in the $C=O$ stretching region 1750

$-1650 \mathrm{~cm}^{-1}$ (arrow indicates direction of cooling from $170^{\circ} \mathrm{C}$ to $50^{\circ} \mathrm{C}$ ); (b) peak fitting corresponding to $\mathrm{T}=50^{\circ} \mathrm{C}$; (c) high frequency region, highlighting the $\mathrm{N}-\mathrm{H}$ stretching band, $\mathrm{T}=50^{\circ} \mathrm{C}$. The contribution at lower frequencies $\left(<1650 \mathrm{~cm}^{-1}\right)$ has been added to facilitate the

fitting procedure. Curves are shifted arbitrarily across the $Y$-axis (IR intensity, a.u.).

We can discriminate contributions to the overall $\mathrm{C}=\mathrm{O}$ stretching region from different populations of carbonyl groups in tAZOi, by fitting the FT-IR spectra to individual peaks, as illustrated in Fig. 2(b). The higher frequency end of the region $\left(\sim 1740 \mathrm{~cm}^{-1}\right)$ comprises mainly contributions from free $\mathrm{C}=\mathrm{O}$ groups, whilst signals at lower wavenumbers correspond to $\mathrm{C}=\mathrm{O}$ groups participating in increasingly stronger hydrogen bonds [39-42]. The peak at around $\sim 1700 \mathrm{~cm}^{-1}$ is normally assigned to $\mathrm{C}=\mathrm{O}---\mathrm{N}-\mathrm{H}$ hydrogen bonds [19, 39], and is coupled with thymine ring vibrations; the contribution at $\sim 1680 \mathrm{~cm}^{-1}$, on the other hand, has been assigned to the $\alpha, \beta$-unsaturated carbonyl group forming strong hydrogen bonds [32]. The prominence of these two latter peaks in Fig. 2(b) is consistent with the preferential assembly of symmetrical tAZOi dimers, Fig. 3, and with the appearance of a signal at 3156 $\mathrm{cm}^{-1}$ related to the stretching vibration of $\mathrm{N}-\mathrm{H}$ groups forming strong hydrogen bonds, Fig.

2(c). Whilst the formation of supramolecular complexes is demonstrated by our current and previous FT-IR analyses [32], the absence of X-ray diffractions at sufficiently low angles seems to discard the formation of large supramolecular aggregates, such as trimers or rosette-like structures. Instead, the XRD of the crystal tAZOi sample shows intense signals in the wide angle region, at spacings around $\sim 5 \AA$ and $4 \AA$, which must be ascribed to structural small repeating units with periodicity in the crystal lattice, such as the azobenzene cores or the thymine heads, as well as first and second order diffractions at smaller angles, which are consistent with the formation of dimers, see also Fig. ESI1. The small changes in the shapes of the FT-IR spectra in Fig. 2(a) confirm that these hydrogen bonds are highly stable with temperature and the performance of tAZOi can be then explained in terms of the hydrogen-bonded complexes. 


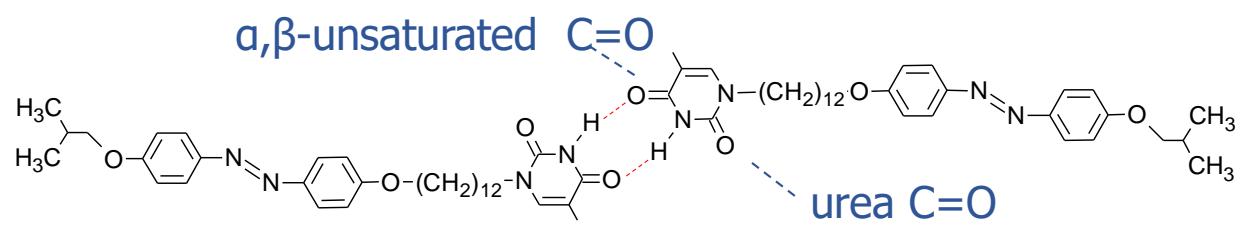

Fig. 3. Schematic sketch of the $t A Z O i$ linear dimer, $(t A Z O i)_{2}$, stabilised by hydrogen-bonding (red dotted lines) between individual tAZOi molecules.

\subsection{Linear Optical Properties of tAZOi}

The linear optical properties of tAZOi are now described, and related to its electronic response. The optical absorption $(A)$ and reflectance $(R)$ spectra of the tAZOi film have been obtained directly from the UV-vis measurement at room temperature, and the results are shown in Fig. 4(a) and Fig. 5(a), respectively. The optical absorption coefficient $(\alpha)$, the refractive index $(n)$, and the extinction coefficient $(k)$, were then calculated using the following relationships [43],

$$
\alpha=\frac{2.303 A}{t}
$$

$$
k=\frac{\alpha \lambda}{4 \pi}
$$

$$
\begin{aligned}
& T=(1-R)^{2} \exp (-\alpha t) \\
& n=\left(\frac{1+R}{1-R}\right)+\sqrt{\left(\frac{4 R}{(1-R)^{2}}-k^{2}\right)}
\end{aligned}
$$

where $A$ is absorbance, $R$ is reflectance, $T$ is transmittance, $\lambda$ is the wavelength of the incident light on the sample, and $t$ is the thickness of the film. The relationship between the optical absorption coefficient and the optical band gap is expressed using the Tauc relationship [44], 


$$
\alpha h v=\alpha_{o}\left(h v-E_{g}\right)^{r}
$$

where $\alpha_{o}$ is an energy-independent constant, $h\left(6.626 \times 10^{-34} \mathrm{~m}^{2} \mathrm{~kg} \cdot \mathrm{s}^{-1}\right)$ is the Planck's constant, $E_{g}$ is the optical band gap, and $r$ is a constant that determines the type of the absorption transition, i.e., $r=0.5$; direct transition, $r=1.5$; direct forbidden transition, $r=2$; indirect transition. From Eq. 5, the following equation was derived,

$$
\frac{d \ln (\alpha h v)}{d h v}=\frac{r}{h v-E_{g}}
$$

By plotting $d \ln (\alpha h v) / d h v$ against $h v$ in the energy range from 2.6 to $3.9 \mathrm{eV}$, see Fig. 4(a), the peak at the highest energy was used to estimate the optical band gap, giving $E_{g} \sim 2.99$ $\mathrm{eV}$. The slope of the $\ln (\alpha h v) v s \ln \left(h v-E_{g}\right)$ plot gives an $r=1.5$ value, see inset in Fig 4(b), indicating that the absorption is a direct forbidden transition. The Tauc plot for the tAZOi film is shown in Fig. 4(c). The intersect of the linear extrapolation of $(\alpha h v)^{1.5}=0$, with a corrected base line, provides the optical band gap, $E_{g}=2.88 \mathrm{eV}$, which represents a $3.8 \%$ deviation respect to the value estimated above.
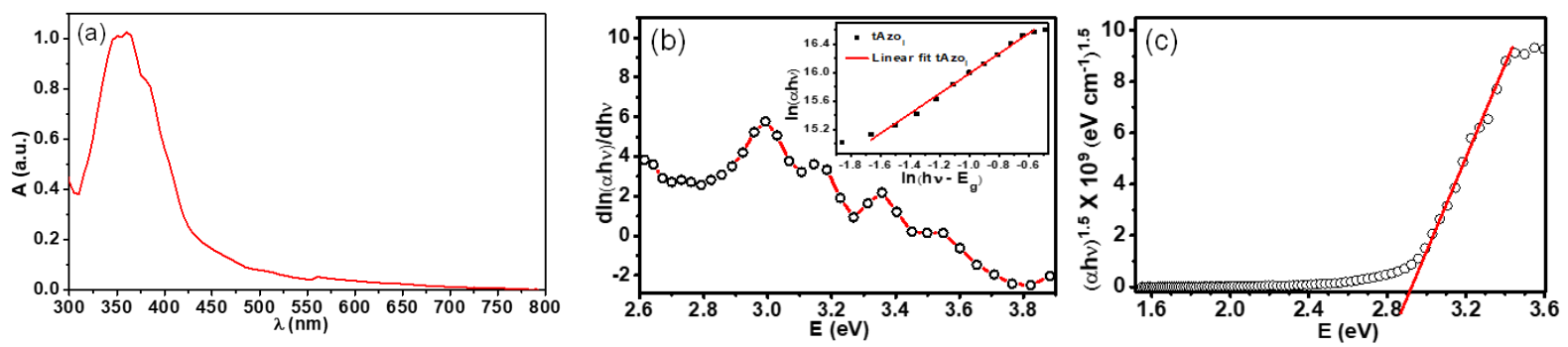

Fig. 4. (a) Absorption spectrum, $A$, and (b) plot of $d \ln (\alpha h v) / d(h v)$ against $h v$, obtained for the tAZOi film. The inset in (b), dln(ahv) vs $\ln \left(h v-E_{g}\right)$, was plotted to determine the constant $r$. (c) Tauc plot of (ahv) ${ }^{1.5} v s$ photon energy (E).

From its reflectance $(R)$ and absorbance $(A)$ spectra, Fig. 5(a) and Fig. 4(b), respectively, it is obvious that tAZOi shows high transmittance at $\lambda>500 \mathrm{~nm}$. More specifically, the extinction coefficient $(k)$ measures the strength of the chemical species absorbing light at a particular wavelength, and depends on the chemical composition and the structure of the material $[45,46]$. The value of $k$ is the highest in the UV region $(\sim 350 \mathrm{~nm})$ and decreases with increasing wavelength from 380 to 800 nm, see Fig. 5(b).

Fig. 5(c), on the other hand, shows the dispersion curve of the refractive index, $n$, in the 300 - $800 \mathrm{~nm}$ wavelength range. The curve shows normal dispersion at $\lambda>420 \mathrm{~nm}$, and anomalous dispersion at $\lambda<420 \mathrm{~nm}$. One peak is observed at $3.757 \mathrm{eV}$, corresponding to the energy transition of the electronic interband UV-absorption at $\lambda \sim 320 \mathrm{~nm}$. 

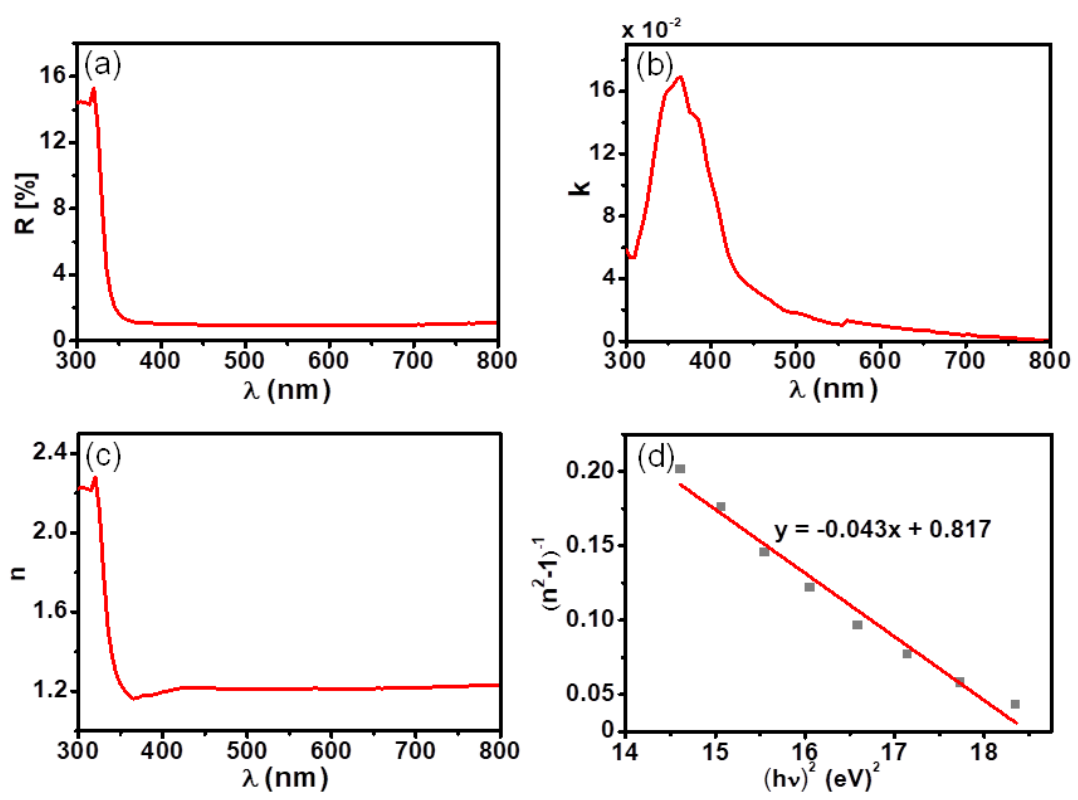

Fig. 5. (a) The spectral distribution of reflectance, $R$; (b) extinction coefficient, $k$, and (c) refractive index, $n$, as a function of the wavelength for the tAZOi film; (d) displays the variation of $\left(n^{2}-1\right)^{-1}$ against $(h v)^{2}$.

The mean refractive index in the wavelength range of $450-800 \mathrm{~nm}$ is $n=1.208$. Using the single oscillator model, Wemple-DiDomenico relationship [47], the measured refractive index was analysed in the $\lambda>300 \mathrm{~nm}$ region to obtain the oscillator parameters, $E_{d}$ and $E_{o}$, according to Eq. 7 ,

$$
\frac{1}{\left(n^{2}-1\right)}=\frac{E_{o}}{E_{d}}-\frac{E^{2}}{E_{o} E_{d}}
$$

where $E$ is the photon energy, $E_{o}$, the average excitation energy for electronic transition, and $E_{d}$, the dispersion energy, which measures the average strength of interband optical transitions or the oscillator strength ${ }^{48}$. The variation of $\left(n^{2}-1\right)^{-1}$ against $(h v)^{2}$ for the tAZOi film was plotted in Fig. 5(d), and the oscillator strength parameters were calculated from this graph, including $E_{o}$ (as the $\mathrm{Y}$-intercept) and $E_{d}$ (as the slope of the curve). These values were then used to calculate the $M_{-1}$ and $M_{-3}$ moments, according to $[49,50]$,

$$
\begin{gathered}
E_{o}^{2}=\frac{M_{-1}}{M_{-3}} \\
E_{d}^{2}=\frac{M_{-1}^{3}}{M_{-3}}
\end{gathered}
$$

and the static refractive index $\left(n_{o}\right)$ was calculated from $n^{2}(h v=0)=n_{o}=\frac{E_{d}}{E_{o}}+1$. 
The $n_{o}, \varepsilon_{\infty}, E_{o}, E_{d}, E_{g}, M_{-1}$ and $M_{-3}$ values calculated for tAZOi are displayed in Table 1, and its linear optical constants are comparable to other organic-based thin films reported in the literature $[48,50]$. It is worth noting that $\varepsilon_{\infty}$ is almost constant in the spectral field of the visible range. The oscillator energy $E_{o}$ is an average energy gap and is in close approximation, $E_{o} \approx 1.5 E_{g}$, with the optical bandgap, as suggested by the single oscillator model, and we can then argue that the Wemple-DiDomenico model describes well the behaviour of tAZOi layered on the ITO substrate. The $M_{-1}$ and $M_{-3}$ moments determine the average bond strength and are related to macroscopic quantities like effective dielectric constant and effective number of valence electrons [48]. The small $M_{-1}$ values of $t A Z O i$ indicate lower $E_{d}$ values with respect to $E_{o}$, and thus relate to weaker interband transitions.

Table 1. Optical parameters of tAZOi and other organic-based thin films.

\begin{tabular}{|c|c|c|c|}
\hline Optical parameters & tAZOi & $\begin{array}{c}\text { Azo dye } \\
{[48]}\end{array}$ & $\begin{array}{c}\text { (Ni)-2-[4-(3-methyl-3- } \\
\text { mesitylcyclobutyl) thiazole- } \\
\text { 2-yl]-hydrazonometyl]- } \\
\text { benzene-2,5-diol thin film } \\
{[50]}\end{array}$ \\
\hline$n_{o}$ & 1.49 & 1.63 & - \\
\hline$\varepsilon_{\infty}$ & 2.22 & 2.66 & 5.80 \\
\hline$E_{o}(e V)$ & 4.36 & 5.34 & 1.598 \\
\hline$E_{d}(e V)$ & 5.33 & 8.90 & 8.044 \\
\hline$E_{o} E_{d}(e V)^{2}$ & 23.24 & 47.52 & 12.85 \\
\hline$E_{g}(e V)$ & 2.88 & 3.3 & 2.13 \\
\hline$E_{o} / E_{g}$ & 1.51 & 1.61 & 0.75 \\
\hline$M_{-1}(e V)^{2}$ & 1.22 & 1.66 & 1.77 \\
\hline$M_{-3}(e V)^{2}$ & 0.064 & 0.0583 & 0.69 \\
\hline
\end{tabular}

The absorption spectra of the tAZOi thin film, before and after exposure to UV light, are shown in Fig. 6(a). The absorption peak in the low wavelength region ( $262 \mathrm{~nm})$ is attributed to the $\pi-\pi^{*}$ electronic transition, associated with the azobenzene E-isomer, whilst the weak broad peak at the longer wavelength $(\sim 450 \mathrm{~nm})$, is attributed to the symmetric forbidden $n-\pi^{*}$ transition [7]. These results are in great agreement with our previous findings, but, compared with the absorption spectra obtained in tetrahydrofuran solution [18], our peaks are broader and suggest the formation of molecular aggregates in the film. More specifically, we can see contributions at $\sim 385 \mathrm{~nm}$, associated to the so-called J-aggregates 
(head-to-tail); at $\sim 362 \mathrm{~nm}$, associated to non-aggregated azobenzene; and at $\sim 342 \mathrm{~nm}$, which we associate to the so-called $\mathrm{H}$-aggregates (head-to-head, parallel) $[7,18]$. Whilst the peak maximum before UV exposure suggests a prevalence of non-aggregated tAZOi molecules ( $362 \mathrm{~nm})$, it is clear that the azobenzene aggregates may have an influence on the material response to external stimuli.

Upon irradiating the film at $365 \mathrm{~nm}$, the intensity of the absorption band at $\sim 360 \mathrm{~nm}$ decreases abruptly accompanied with a slight increase in the intensity of the $450 \mathrm{~nm}$ region, indicating $E$-to- $Z$ photo-isomerisation of the azobenzene units. When the samples are kept in the dark and are subsequently measured at different relaxation times, the intensity of the $\pi-\pi^{*}$ band recovers, whilst the $n-\pi^{*}$ band declines. The maximum UV absorbance ( 360 $\mathrm{nm}$ ) is plotted as a function of the relaxation time in Fig. 6(b), showing that the original UV absorption spectrum is recovered after 48 hours, in good agreement with the dynamic behaviour of other azo-based films [19]. We also note that, on irradiation, the $\sim 362 \mathrm{~nm}$ and $\sim 385 \mathrm{~nm}$ contributions seem to decrease to a greater extent than the $\sim 342 \mathrm{~nm}$ peak, suggesting that a smaller amount of $\mathrm{H}$-aggregates undergo $E$-to- $Z$ isomerisation, perhaps due to the stronger intermolecular interactions in parallel arrangements of tAZOi units.
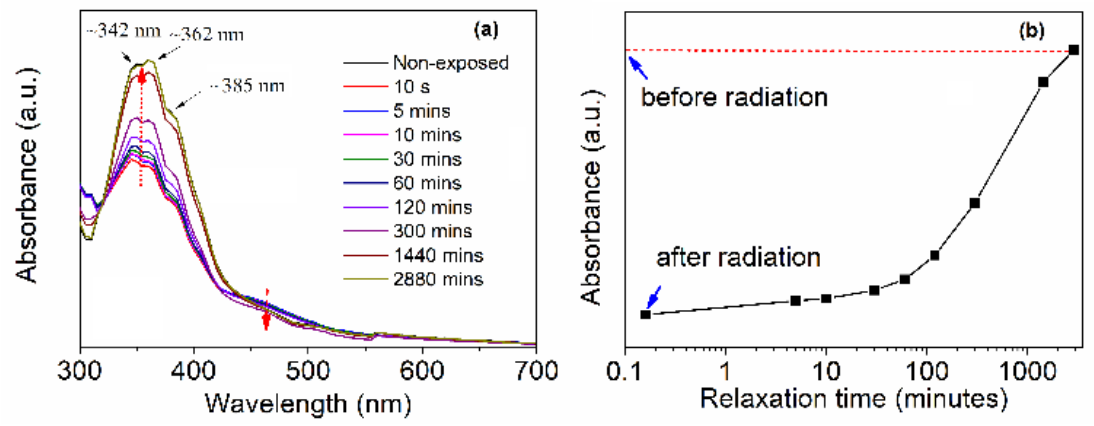

Fig. 6. (a) UV-vis spectra of tAZOi measured at room temperature obtained on a film cast on quartz substrate; red dotted arrows indicate the $Z$-to- $E$ thermal relaxation with time, after exposure to UV light and while keeping the samples in the dark; (b) the exponential increase of absorption intensity at $360 \mathrm{~nm}$, over relaxation time.

\subsection{Electrochemical properties of tAZOi}

The electrochemical properties of tAZOi were investigated by cyclic voltammetry, CV, and more specifically, they were measured on tAZOi crystals immobilised on the surface of an ITO electrode, in $0.1 \mathrm{M} \mathrm{KCl}$ solution. Fig. 7 shows the results, which are summarised in Table 2. From these CV results and the UV-vis absorption spectra, we have calculated the highest occupied and lowest unoccupied molecular orbital energy levels (HOMO and LUMO, respectively), 


$$
E_{\text {HOMO } / \text { LUMO }}=-e\left[E_{O X / R E D}+4.4\right]
$$

where $E_{O X}$ and $E_{R E D}$ are the onset values of the oxidation and reduction potential curves, respectively, and $e$ is the electron charge. The external standard potential of the ferrocene/ferrocenium ion couple is $4.4 \mathrm{eV}$, and the optical band gap is determined from the absorption edge, with $E_{g}=2.81 \mathrm{eV}$. The oxidation peak of the sample appears at approximately $1.12 \mathrm{~V}$, with the onset potential around $0.93 \mathrm{~V}$. Thus, the HOMO energy level was estimated at $\approx-5.33 \mathrm{eV}$, and the LUMO energy level can be calculated at $\approx-2.53 \mathrm{eV}$, by considering the UV-vis absorption spectrum and the HOMO level.

Differences between the energy gaps obtained from UV-vis and CV analyses are common, and can be explained by the presence of deep electronic states in the HOMO and LUMO gap, which shift the oxidation and reduction process [43]. Fig. 7(b) illustrates the energy level diagram of the device under forward bias, showing the electron affinity and ionisation potential of tAZOi. The energy barrier between the HOMO level of TAZOi and the work function of ITO is much smaller, $\Phi_{\text {ITO }}=0.95 \mathrm{eV}$, than the electron injection energy barrier between the work function of the Al electrode and the LUMO energy level, $\Phi_{A l}=1.55 \mathrm{eV}$, refer to Fig. 7(b). Hence, tAZOi behaves like a p-type material and the conduction process in the memory device is dominated by hole injection.

Table 2. Optoelectronic properties and energy levels of tAZOi.

\begin{tabular}{cccccc}
\hline $\begin{array}{c}\text { UV-vis } \lambda_{\max } \\
(\mathrm{nm})\end{array}$ & $\begin{array}{c}\mathrm{E}_{\mathrm{g}}{ }^{\mathrm{a}} \\
(\mathrm{eV})\end{array}$ & $\begin{array}{c}\mathrm{E}_{\text {ox }} \text { (onset) } \\
(\mathrm{V})\end{array}$ & $\begin{array}{c}\text { HOMO } \\
(\mathrm{eV})\end{array}$ & $\begin{array}{c}\text { LUMO } \\
(\mathrm{eV})\end{array}$ & $\begin{array}{c}\mathrm{E}_{\mathrm{g}}{ }^{\mathrm{b}} \\
(\mathrm{eV})\end{array}$ \\
\hline 360 & 2.88 & 0.94 & -5.3 & -2.53 & 2.80 \\
\hline a obtained by UV-vis spectrophotometry. & & & \\
b obtained by cyclic voltammetry.
\end{tabular}



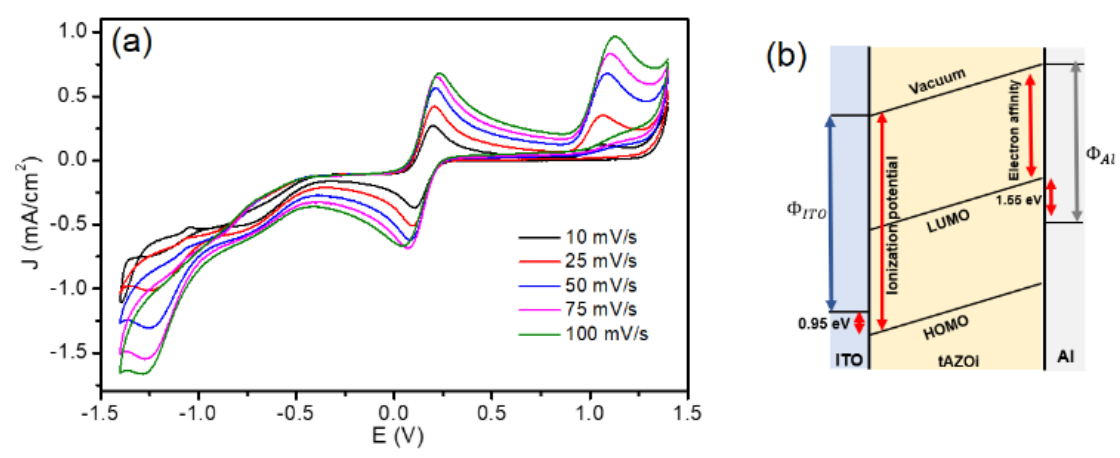

Fig. 7. (a) Cyclic voltammogram (CV) of the tAZOi film on an ITO substrate in $0.1 \mathrm{M} \mathrm{KCl}$ solution as a supporting electrolyte; (b) schematic energy level diagram for the ITO/tAZOi/AI device under forward bias where $\Phi_{\text {ITO }}$ and $\Phi_{A l}$ represent the work function of ITO and Al, respectively.

\subsection{Dielectric properties of the device}

We now investigate the dielectric response of tAZOi as a function of UV exposure $(\lambda=365$ $\mathrm{nm})$, by measuring the complex permittivity, $\varepsilon^{*}$, of the device at room temperature,

$$
\varepsilon^{*}=\varepsilon^{\prime}-i \varepsilon^{\prime \prime}
$$

where $\varepsilon^{\prime}$ and $\varepsilon^{\prime \prime}$ are the real and imaginary dielectric permittivity, respectively, and $i$ is the imaginary unit, $\sqrt{-1}$. According to Fig. 8, permittivity increases upon UV light exposure, and this effect is more acute at lower frequencies, when the movement of charge carriers in the sample becomes more important due to the prevalence of Ohmic conduction contributions. This result confirms that UV irradiation promotes an excess of charge carriers in the tAZOi film. The dielectric permittivity reflects re-orientations of dipoles with respect to the principal molecular axis, see for example [21]. When the sample is irradiated with UV light, E-to- $Z$ isomerisation results in a larger concentration of $Z$ isomers, and the non-linear geometry results in a net dipole moment different to zero, $\vec{\mu}$, see Fig. 1 . If the molecules were to be aligned respect to the device surface, our results indicate that the variation in the pretilt angle may benefit electronic transfer perpendicular to the boundaries, resulting in higher dielectric permittivity.

It is worth noting that the complex permittivity fully recovered the original value after four hours, and through eventually all the frequency range, which highlights the potential of this capacitor in various electronic systems requiring different frequencies. 

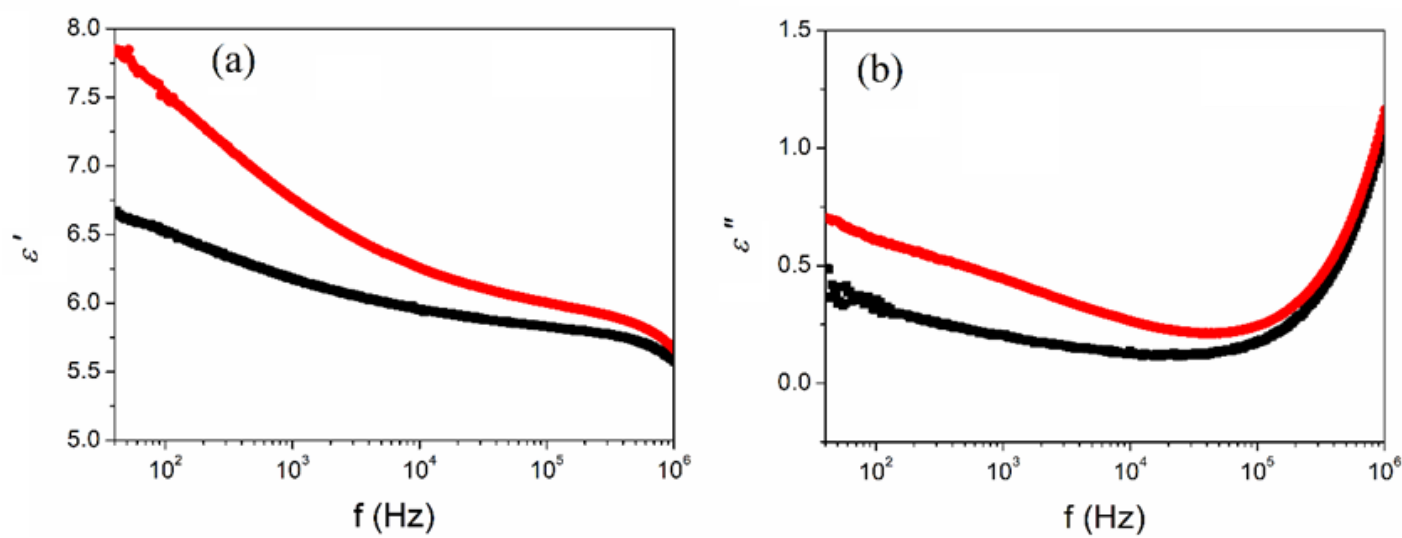

Fig. 8. Frequency dependence of the complex permittivity of tAZOi, $\varepsilon^{*}$, measured at room temperature: (a) real dielectric permittivity, $\varepsilon^{\prime}$; (b) imaginary dielectric permittivity, $\varepsilon^{\prime \prime}$; before (घ) and after (O) UV exposure.

\subsection{Memory effect of the device}

Fig. 9 shows the current-voltage (I-V) curve of the ITO/tAZOi/AI memory device, in log scale. The $I-V$ hysteresis loop is found to be more symmetric about the origin and an intrinsic resistance memory effect of the sample is evident from this reproducible $I-V$ profile. The first sweep was 0 to $-6 \mathrm{~V}$, during which the device shows a sharp increase in the injection current from $10^{-6} \mathrm{~A}$ to $10^{-3} \mathrm{~A}$, at $-4 \mathrm{~V}$. This is the indication of the device transition from low conductance to high conductance state, known as the forming process, and the high (negative) value can be related to the formation of aggregates observed in the UV-vis response. Following the transition, the device was in $\mathrm{ON}$ state even after bias interruption (1 $\min )$, as shown by sweep 2 . The device seems to be in low conductance state during voltage sweeping from 0 to $+6 \mathrm{~V}$, as indicated by sweep 3 . This process with negative SET and positive RESET operation is known as clockwise bipolar resistive switching [35, 51]. High conductance $\mathrm{ON}$ state, $\mathrm{R}_{(\mathrm{ON}) \text { forward }}=1.6 \mathrm{k} \Omega$, and low conductance OFF state, $\mathrm{R}_{(\mathrm{OFF})}$ forward $=4 \mathrm{M} \Omega$, were observed with ON/OFF current ratio $\sim 10^{4}$. An additional DC endurance test was conducted on the memory device and the result is shown in the inset of Fig. 9. The device was successfully switched more than $100 \mathrm{~min}$, without evidence of intermediate states, confirming its stability under constant electrical stress. No degradation was observed during the test. 


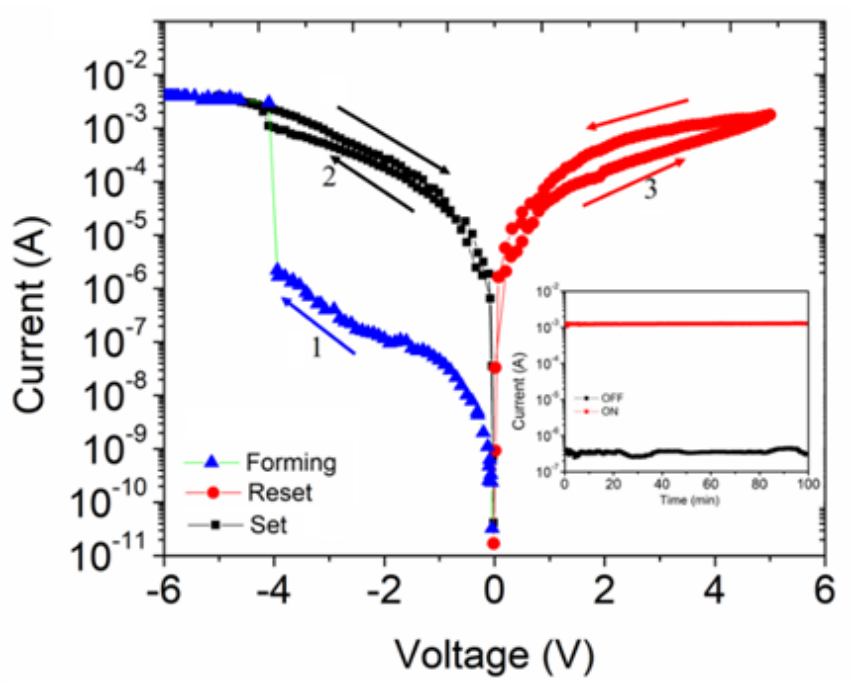

Fig. 9. $L V$ loops of the tAZOi thin film in the device, showing bipolar resistive switching. The inset displays the endurance performance of the device.

Current conduction depends on the resistivity, carrier mobility, geometry, contact and alignment at the interfaces, resulting in changes in the work functions of the material [52]. According to our UV-vis and CV results, the conduction mechanism in the device is dominated by hole injection (tAZOi is p-type semiconductor). The Al/tAZOi/lTO assembly comprises two immediate interfacial regions: the Al/tAZOi and the tAZOi/ITO contact zones. Al/tAZOi can either behave as an ohmic or Schottky contact. Schottky barrier formation at the $\mathrm{Al} / \mathrm{tAZOi}$ interface is probably favourable, since the work function of $\mathrm{Al}$ is $4.08 \mathrm{eV}$, and tAZOi is $2.88 \mathrm{eV}$, causing a potential energy barrier for electrons at a metal-semiconductor junction $[53,54]$. In some cases, the oxide layer formation at the metal/semiconductor interface may contribute to the resistive switching.

To further elucidate the charge transport mechanism, we now analyse the $I-V$ curves in OFF and $\mathrm{ON}$ states based on various theoretical models. The resistive switching behaviour of the tAZOi device is demonstrated in the log-log scale of $I-V$ curve in Fig. 10. The charge transport inside $\mathrm{tAZOi}$ molecules is controlled by trapping and de-trapping of carriers at both energetic and positional distributions [55]. Fig. 10 shows conduction stages with various slope values. At the high resistance state, holes were injected by the positive potential on the top electrode (ITO) of the device. Initially, when a small voltage is applied, a small current is observed since the injected holes remain confined near the ITO contact. In this case, the carrier density is constant and therefore there is effectively no space charge and the device obeys the Ohm's law (region of slope $~ 1$ ). At higher voltages, the injected carriers spread into regions beyond the ITO contact and the injected carrier density becomes larger. Now, the accumulation of carriers near the electrode results in a space charge build up. Mutual repulsion among the injected charges limits further injection of charge carriers 
into the sample, leading to trap limited space charge limited conduction, SCLC (region of slope 2) [63]. This behaviour is consistent with other reported examples in the literature with similar compounds and device configurations [56-57, 63-64]. When sufficient traps are filled and the device is forced with the voltage, consequently, the device transforms from OFF- to ON-state, thus trap-charge limited current (TCLC) [55-57] conduction is observed (region of slope $\sim 4$ ). Subsequently, almost all the trapping sites in the tAZOi are filled, thus the current increases exponentially with increasing applied voltage (refer to the green line in Fig.10).

Traps provide localised states between the HOMO and LUMO energy bandgap of the molecules. In tAZOi molecules, the thymine group might act as hole trapping sites [58] while the azobenzene groups are enriched with electrons [59]. When the applied voltage is higher than the average energy associated with the traps' density, tAZOi molecules behave as trapfree SCLC because the trapped charges are able to move through the trapped sites through a filament formation; consequently, net current is able to flow from the bottom to top electrodes.

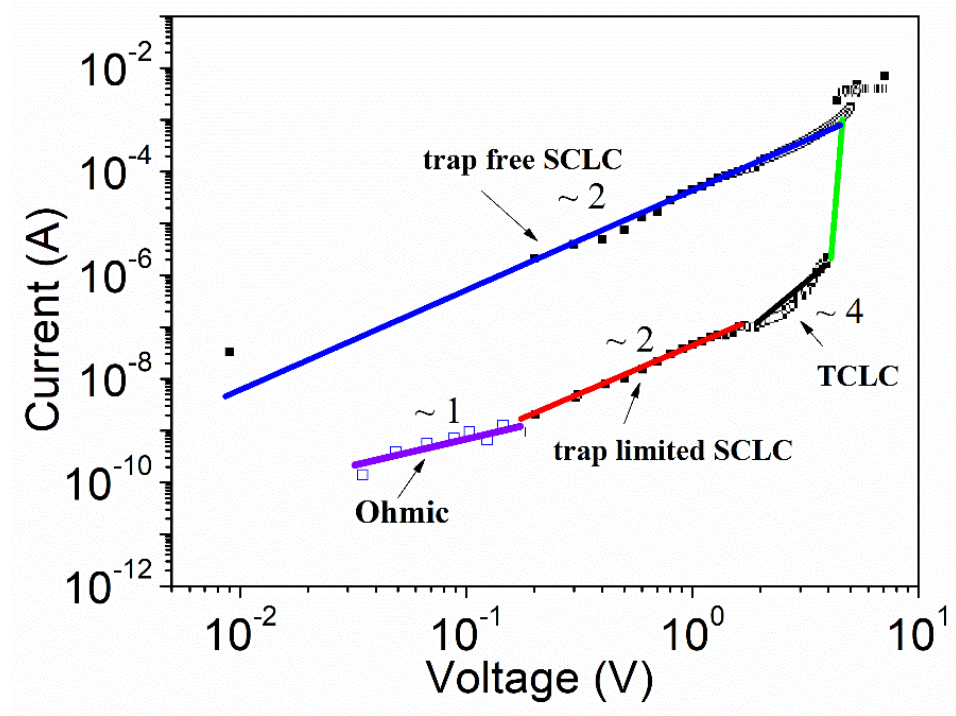

Fig. 10. Experimental and fitted $I-V$ curves for the tAZOi memory device in the OFF and ON states. Charge-transport regions; Ohmic conduction, trap-limited SCLC region, TCLC region, trap-filled voltage $\mathrm{V}_{\mathrm{TFL}}$ region (green line) and trap-free SCLC region.

\subsection{Proposed storage mechanism of the tAZOi memory device}

In order to gain insight into the storage mechanism of tAZOi, a molecular simulation on the basic unit was carried out by DFT/B3LYP/6-311G (d.p.) with the Gaussian 09 program, as shown in Fig.11(a). The charge density for the highest occupied molecular orbital (HOMO) 
and the lowest unoccupied molecular orbital (LUMO), together with an electrostatic potential map (ESP) of the single tAZOi structure were also calculated and shown in Fig. 11.

The HOMO surface indicates the presence of localised electron density on the electron donor in the ground state and the LUMO surface shows the presence of localised electron density on the electron acceptor in the excited state. The charge separated state upon application of electric fields onto the tAZOi molecular complex can be further elucidated by analysing the HOMO and LUMO surfaces, as shown in Fig. 11(b) and 11(c), respectively. The charge density distribution in the HOMO state is primarily surrounding the two benzene rings in Fig. 11(b) and shows a semi-circled distribution of negative and positive charges covering the rings' surface. Whereby, in the LUMO state, the charge density is distributed more likely in the localised fashion on the rings where it can allow the azobenzenes to act as electron acceptor moieties, see Fig. 11(c).

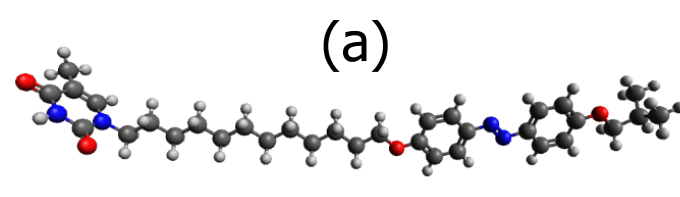

(c)

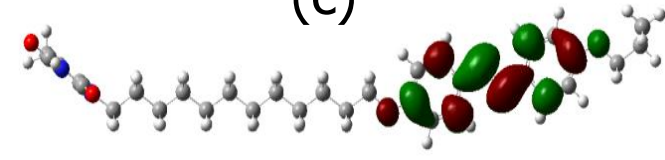

(b)

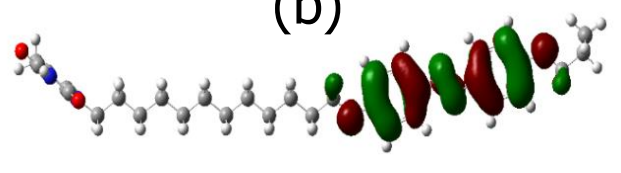

(d)

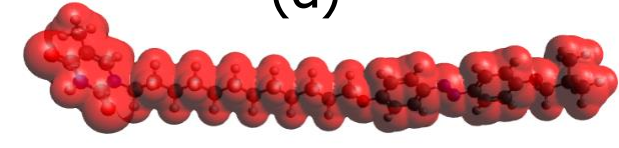

Fig. 11. (a) E-isomer (trans) tAZOi molecule after optimization; (b) HOMO, (c) LUMO, and (d) electrostatic potential map on the molecular surface (ESP).

The HOMO-LUMO energy gap, $\mathrm{E}_{\text {номо-Lumo }}\left(\mathrm{E}_{g}\right)$, obtained for $\mathrm{AZZO}$ from the DFT calculation is about $2.6 \mathrm{eV}$, and this is in good agreement with the UV-vis and CV experimental value of $2.8 \mathrm{eV}$ reported above. Additionally, from our UV-vis and CV results, we found that tAZOi has characteristics of $p$-type semiconductor and therefore the conduction mechanism in the device is highly dominated by hole injection. However, the lower hole-injection barrier of Al/tAZOi/ITO device indicates that the holes could be efficiently injected into the HOMO orbital of tAZOi.

When the electron density localises on the HOMO orbital, the tAZOi memory device is in the OFF state. Upon applying external electric fields with sufficient energy, electrons in the ground state can transit to the various excited states and a charge-transfer (CT) interaction can occur between the electron donor moieties and the electron acceptor moieties. Since the azobenzene group acts like an electron acceptor, the majority of the charge-traps are filled with electrons and a "trap-free" environment with higher charge-carrier mobility is 
created. Moreover, this allows forming a stable CT that facilitates the memory device to transit from the OFF state to the ON state. Consequently, the excitation of the electron donor (the transition of $\pi-\pi^{*}$ type) with the average excitation energy for electronic transition (which is $E_{0} \sim 4.36 \mathrm{eV}$, refer to Table 1 ) can occur. This suggests that the HOMO of the molecule may interact with the LUMO of its neighbouring molecule for charge transfer.

Additionally, the electrostatic potential map of tAZOi, shown in Fig. 11(d), reveals that the surface has a continuous positive ESP (in red) along the flexible alkyl spacer, $\left(\mathrm{CH}_{2}\right)_{12}$, upon applying the electric field, indicating that the charge carriers can migrate from donor to acceptor regions. Considering that thymine is a moderate electron donor [58], whilst azobenzene acts as electron acceptor [59], during the charge transition from HOMO to LUMO, the electron density underwent a minor shift from donor to acceptor sides, implying that it would be easy to return to the pristine state once the electric field is removed [60]. Based on these results, we have sketched in Fig. 12(a) one model to explain the device performance, via double injection [61]. This model is compatible with the formation of $\mathrm{H}$ - and J-aggregates, Fig. 12(b) and 12(c), resp., which we assessed above by UV-vis spectrophotometry, whilst the continuity of charge transport can be explained by a combination of hydrogen-bonding (between thymine heads) and intermolecular aggregation (between azobenzene units).

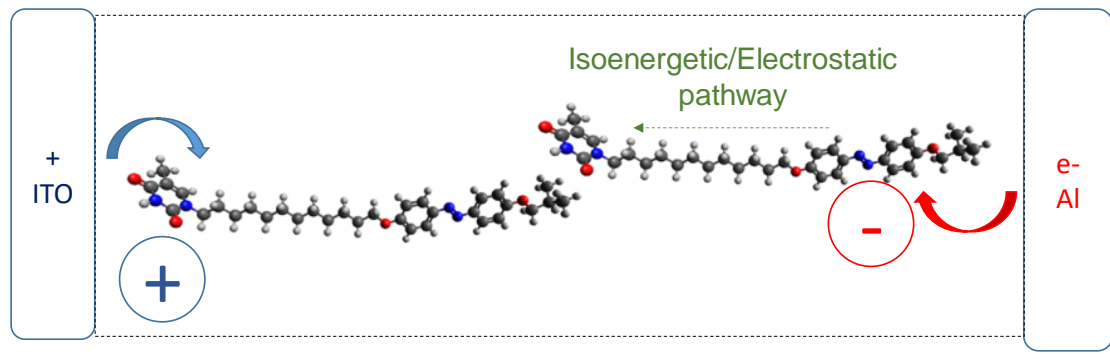

(a)

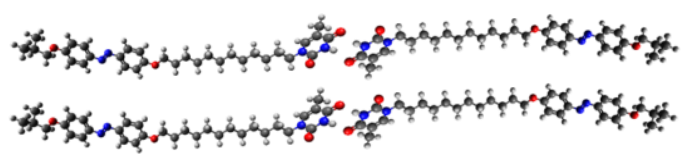

(b)

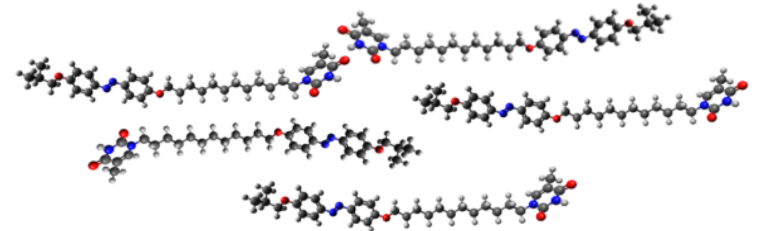

(c)

Fig. 12. (a) Model for charge transfer with double injection; sketches of $(\mathrm{tAZOi})_{2}$ dimers including (b) $\mathrm{H}$-aggregates and (c) J-aggregates.

Even though photoisomerization normally tends to disrupt order in crystalline and liquid crystalline systems due to a reduction in shape anisotropy caused by the presence of the less linear cis linkage [11,62], under our proposed model, the formation of the $Z$ isomer after UV irradiation could facilitate electronic transfer by two-fold. On the one hand, the energy 
gap between the HOMO/LUMO states for the $Z$ isomer of tAZOi are much smaller than for the $E$ isomer (see Fig. 13). On the other hand, the adoption of bent-like geometries by excited tAZOi terminations (LUMO) may facilitate interactions with neighbouring molecules and ultimately electronic transfer with $\mathrm{HOMO}$ regions, reducing the effect of potential $\mathrm{e}^{-}$traps formed in the aggregates $[63,64]$. This is consistent with our dielectric results, which show an increase of $\varepsilon$ " under UV irradiation, and could also explain the low $\mathrm{M}_{-1}$ values obtained above, which denote weaker interband transitions in the single oscillator model.

(a)

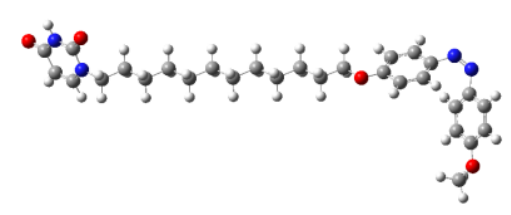

(c)

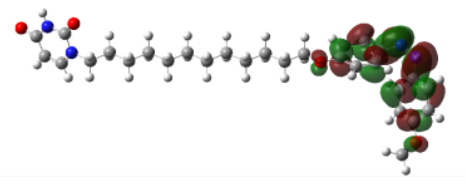

(b)

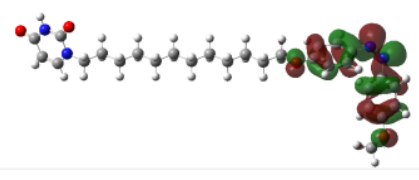

(d)

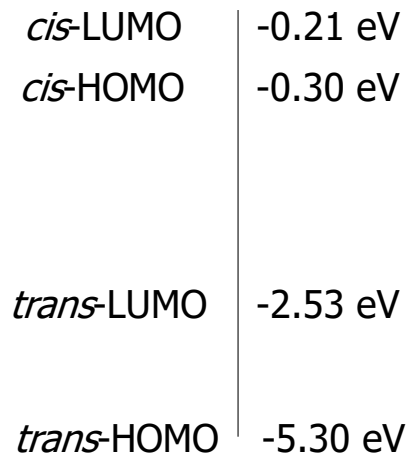

Fig. 13. (a) Z (cis) isomer of the tAZOi molecule after optimization; (b) HOMO, (c) LUMO, and (d) electrostatic potential map on the molecular surface (ESP).

\section{Conclusions}

tAOZi exhibits very promising properties as a light-responsive component in memory devices, based on its electrooptical response. The Al/tAZOi/lTO device has shown lowresistance and high DC endurance and the transition between ON and OFF states relies on p-type conduction, which could be assisted by the hydrogen bonds formed between the thymine units acting as electron donors. According to the isoenergetic surfaces calculated by DFT, charge can be then transferred via the flexible alkyl chains to the azobenzene groups at the molecular terminations, which then act as electron acceptors.

UV irradiation promotes $E$-to- $Z$ photoisomerisation of the azobenzene groups in $\mathrm{tAZOi}$, and the increase in the dielectric constant (and conductivity) is explained by: (i) the high energy of the HOMO and LUMO levels of the $Z$ isomer (and the low HOMO/LUMO energy barrier); and (ii) its capacity for charge transfer due to the change in geometrical geometry. These features will be further explored in the future to obtain new azo-materials that can act as reversible photo-switches for memory devices and energy harvesters, using hydrogenbonding to assemble different functionalities. We will also investigate the role of the 
azobenzene aggregates on charge transfer, and the effect of local ordering in liquid crystalline phases on the performance of photo-electric memory devices.

\section{Conflicts of interest}

There are no conflicts to declare.

\section{Acknowledgements}

TSV acknowledges financial support from the Ministry of Higher Education of Malaysia through the Fundamental Research Grant Scheme [FP079-2018A]. AR acknowledges Ministerio de Economía y Competitividad (MINECO) for his PhD grant BES-2015-071235, under the project MAT2014-55205-P. VMA acknowledges the University Malaya for the grant RF004B-2018. AMF would like to thank the Royal Academy of Engineering, U.K., for the grant NRCP1516/4/61 (Newton Research Collaboration Programme), the University of Aberdeen, for the award of the grant SF10192, the Carnegie Trust for the Universities of Scotland, for the Research Incentive Grant RIG008586, the Royal Society and Specac Ltd., for the Research Grant RGS $\backslash R 1 \backslash 201397$, and the Royal Society of Chemistry for the award of a mobility grant (M19-0000). AMF and TSV further acknowledge University Malaya for travelling support.

\section{References}

[1] D. Bléger, S. Hecht, Visible-Light-Activated Molecular Switches, Angewandte Chemie International Edition, 54 (2015) 11338-11349.

[2] K. Matsuda, M. Irie, Diarylethene as a photoswitching unit, Journal of Photochemistry and Photobiology C: Photochemistry Reviews, 5 (2004) 169-182.

[3] E. Hadjoudis, I.M. Mavridis, Photochromism and thermochromism of Schiff bases in the solid state: structural aspects, Chemical Society Reviews, 33 (2004) 579-588.

[4] S. Jan van der Molen, P. Liljeroth, Charge transport through molecular switches, Journal of Physics: Condensed Matter, 22 (2010) 133001.

[5] A. Bubnov, M. Cigl, N. Sedláčková, D. Pociecha, Z. Böhmová, V. Hamplová, Selfassembling behaviour of new functional photosensitive cinnamoyl-based reactive mesogens, Liquid Crystals, (2020) 1-16.

[6] A. Natansohn, P. Rochon, Photoinduced Motions in Azo-Containing Polymers, Chemical Reviews, 102 (2002) 4139-4176.

[7] G.S. Kumar, D.C. Neckers, Photochemistry of azobenzene-containing polymers, Chemical Reviews, 89 (1989) 1915-1925.

[8] H.M.D. Bandara, S.C. Burdette, Photoisomerization in different classes of azobenzene, Chemical Society Reviews, 41 (2012) 1809-1825.

[9] A.A. Beharry, G.A. Woolley, Azobenzene photoswitches for biomolecules, Chemical Society Reviews, 40 (2011) 4422-4437. 
[10] K. Ichimura, Photoalignment of Liquid-Crystal Systems, Chemical Reviews, 100 (2000) 1847-1874.

[11] D.A. Paterson, J. Xiang, G. Singh, R. Walker, D.M. Agra-Kooijman, A. Martínez-Felipe, M. Gao, J.M.D. Storey, S. Kumar, O.D. Lavrentovich, C.T. Imrie, Reversible Isothermal Twist-Bend Nematic-Nematic Phase Transition Driven by the Photoisomerization of an Azobenzene-Based Nonsymmetric Liquid Crystal Dimer, Journal of the American Chemical Society, 138 (2016) 5283-5289.

[12] I. Chaganava, B. Kilosanidze, G. Kakauridze, L. Oriol, M. Piñol, A. Martinez-Felipe, Induction of the vector polyphotochromism in side-chain azopolymers, Journal of Photochemistry and Photobiology A: Chemistry, 354 (2018) 70-77.

[13] S.M. Alauddin, N.F.K. Aripin, T.S. Velayutham, I. Chaganava, A. Martinez-Felipe, The role of conductivity and molecular mobility on the photoanisotropic response of a new azopolymer containing sulfonic groups, Journal of Photochemistry and Photobiology A: Chemistry, 389 (2020) 112268.

[14] A. Bobrovsky, K. Mochalov, D. Solovyeva, V. Shibaev, M. Cigl, V. Hamplová, A. Bubnov, Laser-induced formation of "craters" and "hills" in azobenzene-containing polymethacrylate films, Soft Matter, 16 (2020) 5398-5405.

[15] A. Bobrovsky, V. Shibaev, M. Cigl, V. Hamplová, D. Pociecha, A. Bubnov, Azobenzenecontaining LC polymethacrylates highly photosensitive in broad spectral range, Journal of Polymer Science Part A: Polymer Chemistry, 54 (2016) 2962-2970.

[16] A. Bobrovsky, V. Shibaev, A. Piryazev, D.V. Anokhin, D.A. Ivanov, O. Sinitsyna, V. Hamplova, M. Kaspar, A. Bubnov, Photo-Orientation Phenomena in Photochromic Liquid Crystalline Azobenzene-Containing Polymethacrylates with Different Spacer Length, Macromolecular Chemistry and Physics, 218 (2017) 1700127.

[17] A. Bobrovsky, V. Shibaev, M. Cigl, V. Hamplová, P. Dorovatovskii, B. Ostrovskii, A. Bubnov, The effect of spacer and alkyl tail lengths on the photoorientation processes in amorphousized films of azobenzene-containing liquid crystalline polymethacrylates, Liquid Crystals, 47 (2020) 377-383.

[18] A. Concellón, E. Blasco, A. Martínez-Felipe, J.C. Martínez, I. Šics, T.A. Ezquerra, A. Nogales, M. Piñol, L. Oriol, Light-Responsive Self-Assembled Materials by Supramolecular Post-Functionalization via Hydrogen Bonding of Amphiphilic Block Copolymers, Macromolecules, 49 (2016) 7825-7836.

[19] L. Vanti, S. Mohd Alauddin, D. Zaton, N.F.K. Aripin, M. Giacinti-Baschetti, C.T. Imrie, A. Ribes-Greus, A. Martinez-Felipe, lonically conducting and photoresponsive liquid crystalline terpolymers: Towards multifunctional polymer electrolytes, European Polymer Journal, 109 (2018) 124-132.

[20] A. Martinez-Felipe, Z. Lu, P.A. Henderson, S.J. Picken, B. Norder, C.T. Imrie, A. RibesGreus, Synthesis and characterisation of side chain liquid crystal copolymers containing sulfonic acid groups, Polymer, 53 (2012) 2604-2612.

[21] B. Robles-Hernández, N. Sebastián, M.R. de la Fuente, D.O. López, S. Diez-Berart, J. Salud, M.B. Ros, D.A. Dunmur, G.R. Luckhurst, B.A. Timimi, Twist, tilt, and orientational order at the nematic to twist-bend nematic phase transition of 1",9"-bis(4-cyanobiphenyl4'-yl) nonane: A dielectric, ${ }^{2}$ H NMR, and calorimetric study, Physical Review E, 92 (2015) 062505.

[22] Z. Li, M. Wang, H. Li, J. He, N. Li, Q. Xu, J. Lu, Rewritable ternary data storage devices based on polymethacrylate containing pendent azobenzene-naphthalene with the combined effects of conformation change and charge traps, Journal of Materials Chemistry C, 5 (2017) 8593-8598.

[23] L. Zhou, Q. Liu, X. Lv, L. Gao, S. Fang, H. Yu, Photoinduced triple shape memory polyurethane enabled by doping with azobenzene and GO, Journal of Materials Chemistry C, 4 (2016) 9993-9997.

[24] D. Gindre, A. Boeglin, A. Fort, L. Mager, K.D. Dorkenoo, Rewritable optical data storage in azobenzene copolymers, Opt. Express, 14 (2006) 9896-9901. 
[25] J. Royes, C. Provenzano, P. Pagliusi, R.M. Tejedor, M. Piñol, L. Oriol, A Bifunctional Amorphous Polymer Exhibiting Equal Linear and Circular Photoinduced Birefringences, Macromolecular Rapid Communications, 35 (2014) 1890-1895.

[26] M. Lomba, L. Oriol, C. Sánchez, A new photoimaging system based on a trisdiazonium salt as a photocrosslinker for sulfonated polyelectrolytes, European Polymer Journal, 45 (2009) 1785-1790.

[27] C. Berges, L. Oriol, M. Piñol, C. Sánchez-Somolinos, R. Alcalá, Blends of an azomethacrylic block copolymer for volume holographic storage using low energy $10 \mathrm{~ms}$ light pulses, Optical Materials, 35 (2013) 1095-1098.

[28] P. Forcén, L. Oriol, C. Sánchez, F.J. Rodríguez, R. Alcalá, S. Hvilsted, K. Jankova, Volume holographic storage and multiplexing in blends of PMMA and a block methacrylic azopolymer, using $488 \mathrm{~nm}$ light pulses in the range of $100 \mathrm{~ms}$ to $1 \mathrm{~s}$, European Polymer Journal, 44 (2008) 72-78.

[29] A. Concellón, E. Blasco, M. Piñol, L. Oriol, I. Díez, C. Berges, C. Sánchez-Somolinos, R. Alcalá, Photoresponsive polymers and block copolymers by molecular recognition based on multiple hydrogen bonds, Journal of Polymer Science Part A: Polymer Chemistry, 52 (2014) 3173-3184.

[30] J. del Barrio, E. Blasco, L. Oriol, R. Alcalá, C. Sánchez-Somolinos, Diblock copolymerazobenzene complexes through hydrogen bonding: Self-assembly and stable photoinduced optical anisotropy, Journal of Polymer Science Part A: Polymer Chemistry, 51 (2013) 1716-1725.

[31] M. Millaruelo, L.S. Chinelatto, L. Oriol, M. Piñol, J.-L. Serrano, R.M. Tejedor, Synthesis and Characterization of Supramolecular Polymeric Materials Containing Azopyridine Units, Macromolecular Chemistry and Physics, 207 (2006) 2112-2120.

[32] A. Martinez-Felipe, F. Brebner, D. Zaton, A. Concellon, S. Ahmadi, M. Piñol, L. Oriol, Molecular Recognition via Hydrogen Bonding in Supramolecular Complexes: A Fourier Transform Infrared Spectroscopy Study, Molecules, 23 (2018) 2278.

[33] S. Lee, W. Kim, K. Yong, Overcoming The Water Vulnerability Of Electronic Devices: A Highly Water-Resistant ZnO Nanodevice With Multifunctionality, Advanced Materials, 23 (2011) 4398-4402.

[34] K.-C. Liu, W.-H. Tzeng, K.-M. Chang, Y.-C. Chan, C.-C. Kuo, Bipolar resistive switching effect in Gd2O3 films for transparent memory application, Microelectronic Engineering, 88 (2011) 1586-1589.

[35] Q.-j. Zhang, J.-h. He, H. Zhuang, H. Li, N.-j. Li, Q.-f. Xu, D.-y. Chen, J.-m. Lu, Ternary Flexible Electro-resistive Memory Device based on Small Molecules, Chemistry - An Asian Journal, 11 (2016) 1624-1630.

[36] J. Shang, W. Xue, Z. Ji, G. Liu, X. Niu, X. Yi, L. Pan, Q. Zhan, X.-H. Xu, R.-W. Li, Highly flexible resistive switching memory based on amorphous-nanocrystalline hafnium oxide films, Nanoscale, 9 (2017) 7037-7046.

[37] M. Frisch, G. Trucks, H.B. Schlegel, G. Scuseria, M. Robb, J. Cheeseman, G. Scalmani, V. Barone, B. Mennucci, G. Petersson, Gaussian 09, revision a. 02, gaussian, Inc., Wallingford, CT, 200 (2009) 28.

[38] T. Yanai, D.P. Tew, N.C. Handy, A new hybrid exchange-correlation functional using the Coulomb-attenuating method (CAM-B3LYP), Chemical Physics Letters, 393 (2004) 5157.

[39] A. Martinez-Felipe, A.G. Cook, J.P. Abberley, R. Walker, J.M.D. Storey, C.T. Imrie, An FT-IR spectroscopic study of the role of hydrogen bonding in the formation of liquid crystallinity for mixtures containing bipyridines and 4-pentoxybenzoic acid, RSC Advances, 6 (2016) 108164-108179.

[40] M.J. Abdy, A. Murdoch, A. Martínez-Felipe, New insights into the role of hydrogen bonding on the liquid crystal behaviour of 4-alkoxybenzoic acids: a detailed IR spectroscopy study, Liquid Crystals, 43 (2016) 2191-2207.

[41] A. Martínez-Felipe, C.T. Imrie, The role of hydrogen bonding in the phase behaviour of supramolecular liquid crystal dimers, Journal of Molecular Structure, 1100 (2015) 429437. 
[42] A. Martínez-Felipe, A.G. Cook, M.J. Wallage, C.T. Imrie, Hydrogen bonding and liquid crystallinity of low molar mass and polymeric mesogens containing benzoic acids: a variable temperature Fourier transform infrared spectroscopic study, Phase Transitions, 87 (2014) 1191-1210.

[43] J.I. Pankove, Optical processes in semiconductors., 18 ed., The Electrochemical Society, 1971.

[44] F. Karipcin, S. Ilican, Y. Caglar, M. Caglar, B. Dede, Y. Şahin, Synthesis, structural and optical properties of novel borylated $\mathrm{Cu}$ (II) and $\mathrm{Co}$ (II) metal complexes of 4benzylaminobiphenylglyoxime, Journal of Organometallic Chemistry, 692 (2007) 24732481.

[45] A.Y. Al-Ahmad, Q.M.A. Hassan, H.A. Badran, K.A. Hussain, Investigating some linear and nonlinear optical properties of the azo dye (1-amino-2-hydroxy naphthalin sulfonic acid-[3-(4-azo)]-4-amino diphenyl sulfone), Optics \& Laser Technology, 44 (2012) 14501455.

[46] M.R. Islam, J. Podder, Optical properties of $\mathrm{ZnO}$ nano fiber thin films grown by spray pyrolysis of zinc acetate precursor, Crystal Research and Technology, 44 (2009) 286292.

[47] S. Wemple, M. DiDomenico Jr, Behavior of the electronic dielectric constant in covalent and ionic materials, Physical Review B, 3 (1971) 1338.

[48] H.A.S. Majeed, A.Y. Al-Ahmad, K.A. Hussain, The Preparation, Characterization and the Study of the Linear Optical Properties of a New Azo, Journal of Basrah Researches (Sciences), 37 (2011) 64-73.

[49] S.H. Wemple, M. DiDomenico, Optical Dispersion and the Structure of Solids, Physical Review Letters, 23 (1969) 1156-1160.

[50] F. Yakuphanoglu, A. Cukurovali, İ. Yilmaz, Determination and analysis of the dispersive optical constants of some organic thin films, Physica B: Condensed Matter, 351 (2004) 53-58.

[51] S.N. Inamdar, P.P. Ingole, S.K. Haram, Determination of Band Structure Parameters and the Quasi-Particle Gap of CdSe Quantum Dots by Cyclic Voltammetry, ChemPhysChem, 9 (2008) 2574-2579.

[52] S. Mitra, S. Chakraborty, K.S.R. Menon, Study of anti-clockwise bipolar resistive switching in Ag/NiO/ITO heterojunction assembly, Applied Physics A, 115 (2014) 1173-1179.

[53] D. Schlettwein, Chapter 3 - Electronic Properties of Molecular Organic Semiconductor Thin Films, in: H.S. Nalwa (Ed.) Supramolecular Photosensitive and Electroactive Materials, Academic Press, San Diego, 2001, pp. 211-338.

[54] F. Yakuphanoglu, M. Kandaz, B.F. Senkal, Current-voltage and capacitance-voltage characteristics of Al/p-type silicon/organic semiconductor based on phthalocyanine rectifier contact, Thin Solid Films, 516 (2008) 8793-8796.

[55] H. Li, N. Li, H. Gu, Q. Xu, F. Yan, J. Lu, X. Xia, J. Ge, L. Wang, Two Different Memory Characteristics Controlled by the Film Thickness of Polymethacrylate Containing Pendant Azobenzothiazole, The Journal of Physical Chemistry C, 114 (2010) 6117-6122.

[56] F.-L. Ye, P.-Y. Gu, F. Zhou, H.-F. Liu, X.-P. Xu, H. Li, Q.-F. Xu, J.-M. Lu, Preparation of homopolymers from new azobenzene organic molecules with different terminal groups and study of their nonvolatile memory effects, Polymer, 54 (2013) 3324-3333.

[57] M.N. Awais, M.N. Shehzad, Prediction of the Current-Voltage Characteristics and the Bipolar Resistive Switching Mechanism in Polymer-Based Sandwiched Structures, Journal of the Korean Physical Society, 75 (2019) 409-414.

[58] B. Pullman, A. Pullman, Electron-Donor and -Acceptor Properties of Biologically Important Purines, Pyrimidines, Pteridines, Flavins, and Aromatic Amino Acids, Proceedings of the National Academy of Sciences of the United States of America, 44 (1958) 1197-1202.

[59] O. Tsutsumi, T. Kitsunai, A. Kanazawa, T. Shiono, T. Ikeda, Photochemical Phase Transition Behavior of Polymer Azobenzene Liquid Crystals with Electron-Donating and Accepting Substituents at the 4,4'-Positions, Macromolecules, 31 (1998) 355-359.

[60] J. Wehner, L. Brombacher, J. Brown, C. Junghans, O. Çaylak, Y. Khalak, P. Madhikar, G. Tirimbò, B. Baumeier, Electronic Excitations in Complex Molecular Environments: 
Many-Body Green's Functions Theory in VOTCA-XTP, J Chem Theory Comput, 14 (2018) 6253-6268.

[61] C. Ye, Q. Peng, M. Li, J. Luo, Z. Tang, J. Pei, J. Chen, Z. Shuai, L. Jiang, Y. Song, Multilevel Conductance Switching of Memory Device through Photoelectric Effect, Journal of the American Chemical Society, 134 (2012) 20053-20059.

[62] H. Yu, H. Liu, T. Kobayashi, Fabrication and Photoresponse of Supramolecular Liquid-Crystalline Microparticles, ACS Applied Materials \& Interfaces, 3 (2011) 13331340.

[63] H. Li, Y. Wen, P. Li, R. Wang, G. Li, Y. Ma, L. Yang, Y. Song, Q. Yang, D. Zhu, A high ON/OFF ratio organic film for photo- and electro-dual-mode recording, Applied Physics Letters, 94 (2009) 163309.

[64] H. Li, Z. Jin, N. Li, Q. Xu, H. Gu, J. Lu, X. Xia, L. Wang, A small-molecule-based device for data storage and electro-optical switch applications, Journal of Materials Chemistry, 21 (2011) 5860-5862. 